\title{
Genotipos de Echinococcus granulosus en hidatidosis humana alrededor del mundo. Revisión sistemática
}

\author{
Echinococcus granulosus genotypes verified in human hydatid disease around the world. Systematic \\ review
}

Carlos Manterola1,2,3, Claudio Rojas 3,4, Armando Totomoch-Serra3 , Nayely García-Méndez y Ángela L. Riffo-Campos³

'Departamento de Cirugía, Universidad de La Frontera. Temuco, Chile.
2Centro de Estudios Morfológicos y Quirúrgicos (CEMyQ), Universidad de La Frontera. Temuco, Chile.
${ }^{3}$ Programa de Doctorado en Ciencias Médicas, Universidad de La Frontera. Temuco, Chile.
${ }^{4}$ Instituto de Salud Pública, Universidad Austral de Chile. Valdivia, Chile.

Carlos Manterola y Claudio Rojas son primeros autores.

Parcialmente financiado por el proyecto DI19-0030, Dirección de Investigación, Universidad de La Frontera.

Conflictos de interés: Ninguno.

PROSPERO ID: CRD42018099827

Recibido: 3 de diciembre de 2019 / Aceptado: 27 de agosto de 2020

\section{Resumen}

Introducción: La evidencia sobre las características genotípicas de la infección por Echinococcus granulosus en humanos es escasa. Objetivo: Desarrollar un resumen de la evidencia disponible respecto a genotipos de E. granulosus verificados en hidatidosis humana en el mundo. Material y Métodos: Revisión sistemática. Se incluyeron artículos relacionados con genotipos de E. granulosus, en humanos, sin restricción de lenguaje ni método de secuenciación; publicados entre 1990-2019. Se realizó una búsqueda sistemática en WoS, EMBASE, MEDLINE, SCOPUS, Trip Database, BIREME, SciELO, LILACS, IBECS y OPS-OMS. Las variables en estudio fueron: año de publicación, país de origen, número de muestras, órganos parasitados, marcador molecular utilizado y genotipo identificado. Se aplicó estadística descriptiva. Resultados: Se identificaron 701 artículos relacionados; 62 cumplieron los criterios de selección, representando 1.511 muestras. La evidencia existente fue publicada entre 1994 y 2019 y proviene principalmente de Irán $(45,2 \%)$. El método de secuenciación más utilizado fue amplificación por reacción de polimerasa en cadena más secuenciación tipo Sanger con genotipificación del gen cox1 $(79,0 \%)$. Los genotipos identificados con mayor frecuencia fueron G1 (49,1\%) y el complejo G1/G3 (32,2\%). Conclusión: Las publicaciones relacionadas con genotipos de E. granulosus en humanos son escasas y heterogéneas. $E g$ G1 representa la mayor parte de la carga global mundial.

Palabras clave: Echinococcus granulosus; echinococcosis; genotipos; haplotipos; epidemiología molecular.

\begin{abstract}
Background: The evidence regarding genotypic characteristics of Echinococcus granulosus infection in humans worldwide is scarce. Aim: To develop a synthesis of the available evidence regarding genotypes of E. granulosus verified in humans worldwide. Methods: Systematic review. Articles related with genotypes of E. granulosus, in humans, without language neither genotyped method restriction, published between 1990-2019 were included. A systematic in WoS, EMBASE, MEDLINE, SCOPUS, Trip Database, BIREME, SciELO, LILACS, IBECS, and PAHO-WHO was carried out. In study variables were year of publication, country, number of samples, host and parasite organs, genotype identified, molecular marker and genes. Descriptive statistics were applied. Results: 701 related articles were identified; 62 fulfilled selection criteria, representing 1,511 samples. The existing evidence was published between 1994 and 2019; and mainly comes from Iran (45.2\%). The most commonly used sequencing method was PCR amplification and Sanger type sequencing with partial or total genotyping of the cox 1 gene. Genotyped method most frequently used was coxl $(79,0 \%)$. Genotypes most frequently identified were G1 and G1/G3 complex (49.1\% and 32.2\%). Conclusions: Publications related to genotypes of $E g$ in humans are scarce, heterogeneous, and presenting differing results. $E g$ G1/G3 accounts for most of the global burden worldwide.

Keyword: Echinococcus granulosus; echinococcosis; genotype; haplotypes; molecular epidemiology.
\end{abstract}

\section{Correspondencia a:}

Carlos Manterola Delgado

carlos.manterola@ufrontera.cl
Claudio Rojas Pincheira

crojas.vet@gmail.com 


\section{Introducción}

L a hidatidosis o equinococosis quística es una zoonosis parasitaria que afecta, tanto a humanos como a otros mamíferos; los que se ven parasitados por la etapa larval del Echinococcus granulosus ${ }^{1}$.

Esta enfermedad que se asocia con la pobreza y malas prácticas de higiene se ha descrito especialmente en comunidades ganaderas; sin embargo, los procesos migratorios han permitido que poblaciones expuestas e infectadas se diagnostiquen incluso en áreas geográficas en las que tradicionalmente no existe o no se reporta. Es una afección prevenible con medidas educativas, pero a pesar de ello, la OMS la considera como una de las 17 denominadas "enfermedades desatendidas"1,2.

Esta zoonosis se distribuye en todo el mundo y es endémica o hiperendémica en algunos países de Asia, América del Sur, Europa del este, y norte de África; y es responsable de la pérdida de alrededor de 3,6 millones de años de vida ajustados por discapacidad (AVAD) a nivel mundial por la infección de seres humanos ${ }^{3,4}$.

En un intento por estudiar el componente genético de E. granulosus, se han analizado genes mitocondriales (cox1, nad1 principalmente) con mayor presencia de polimorfismos, identificando de este modo, grupos de genotipos y algunos haplotipos de E. granulosus ${ }^{5}$. Es así como mediante técnicas de biología molecular basadas en la reacción de polimerasa en cadena (RPC) y análisis de secuencias de $\mathrm{ADN}$, se ha podido caracterizar el complejo de genotipos de este cestodo, denominado E. granulosus sensu lato, el que, para su estudio y clasificación genética, se encuentra compuesto por E. granulosus sensu stricto (G1, G2, y G3), E. equinus (G4), E. ortleppi (G5) y E. canadensis (G6, G7, G8, G9 y G10). Estos grupos presentan variaciones fenotípicas, especificidad por el hospedero intermedio, diferencias en su patogenicidad, antigenicidad, etc. ${ }^{5,6}$. También se han subagrupado como complejo G1/ G3, G4, G5, complejo G6/G7, G8, G9 y G10.

El objetivo de este estudio fue desarrollar un resumen de la evidencia disponible respecto a los genotipos de $E$. granulosus verificados en hidatidosis humana en todo el mundo.

\section{Metodología}

Este estudio fue escrito siguiendo la guía PRISMA ${ }^{7}$ y está registrado como protocolo en PROSPERO (ID: CRD42018099827).

\section{Diseño}

Revisión sistemática (RS).

\section{Criterios de elegibilidad}

Se incluyeron artículos relacionados con genotipos de E. granulosus, en humanos, sin lenguaje ni restricción del método genotipado, publicados entre 1990 y 2019. Se excluyeron artículos de revisión, cartas al editor, registros de hidatidosis con ambigüedades en la información de los genotipos; y estudios donde se incluyó equinococosis alveolar o multilocular.

\section{Fuentes de información}

Se realizó una búsqueda sistemática de literatura relacionada en las siguientes fuentes: WoS, EMBASE, MEDLINE, SCOPUS, Trip Database, BIREME-BVS, SciELO, LILACS, IBECS, y OPS-OMS. Además, se realizó una búsqueda de referencias cruzadas de forma manual.

\section{Búsqueda de la información}

La búsqueda se centró en el período 1990-2019. Se utilizaron los términos $\mathrm{MeSH}$ y palabras libres: "Echinococcus", "Echinococcus granulosus", "Echinococcus granulosus sensu lato", "equinococosis", "quiste hidatídico", "hidatidosis", "genotipo", “cepa", "especie", "secuencia", "marcador molecular" y "gen". Además, se usaron operadores booleanos AND y OR. El reclutamiento de artículos se cerró el 30 de noviembre de 2019. Las búsquedas se adaptaron a cada base de datos e idioma correspondiente. La estrategia de búsqueda para cada fuente de información se resume en la Tabla 1.

\section{Selección de los estudios}

La evaluación de elegibilidad de los artículos primarios fue ejecutada por dos grupos de dos revisores cada cual (CM-CR y AT-NG), los que actuaron de forma independiente y enmascarada. Los desacuerdos entre los grupos de revisores se resolvieron por consenso.

\section{Proceso de recopilación de datos}

Para la extracción de datos, se creó una hoja Excel (Mac Excel, versión 15.24; 2016 Microsoft Corporation ${ }^{\circledR}$ ). Dos autores extrajeron los datos de los estudios incluidos (CM y CR); y otros dos, verificaron los datos extraídos (NG y AT). Los desacuerdos se resolvieron por consenso entre los revisores.

\section{Variables en estudio}

Características de los participantes de los estudios (año de publicación, país, región, número de muestras, hospedero, órgano de origen de las muestras y tipo de diseño del estudio primario); el tipo de intervención (técnica de biología molecular y genes utilizados); medida de resultado (genotipo identificado).

\section{Extracción de datos y variables en estudio}

Se extrajo información de cada estudio incluido, independientemente de que estos fueran de muestras humanas exclusivas, o incluyeran humanos y otros hospederos (en estos casos, se analizaron sólo los datos de las muestras humanas). 


\section{Riesgo de sesgo en los estudios individuales}

Los posibles estudios faltantes se capturaron mediante la búsqueda de referencias cruzadas. Respecto de la validez interna de los estudios primarios, no se evaluaron posibles sesgos, puesto que todos ellos son series de casos.

\section{Medidas de resumen}

Se utilizaron estadísticas descriptivas (porcentajes y cálculo de promedios).

\section{Síntesis de los resultados}

Los documentos identificados en cada fuente de información se filtraron por duplicación entre bases. Luego, los títulos y resúmenes se examinaron aplicando criterios de selección. Finalmente, se realizó un análisis en profundidad de cada uno de los artículos primarios seleccionados, aplicando guías de lectura crítica. Esto permitió organizar la síntesis de la información.

\section{Análisis adicionales}

No se realizaron.

\section{Ética}

Los autores y centros de los estudios primarios utilizados fueron enmascarados.

\section{Resultados}

De la búsqueda en las mencionadas fuentes de información, se recuperaron 701 registros (Tabla 1), a los que se adicionaron 35 obtenidos a partir de la búsqueda de referencias cruzadas. En esta misma etapa, se verificó que 153 eran artículos duplicados entre las diferentes bases de datos consultadas. A continuación, se procedió al análisis de títulos y resúmenes, lo que permitió eliminar 362 registros por no cumplir criterios de inclusión y exclusión. Luego, se procedió al análisis en profundidad de los 221 estudios seleccionados; y a la lectura detallada de éstos, lo que permitió excluir 159 artículos, para quedar finalmente con 62 estudios que cumplían con los criterios de inclusión y exclusión ${ }^{6,8-68}$, los que corresponden al material de análisis de este estudio (Figura 1); y que representan un total de 1.511 muestras. Todos los estudios finalmente seleccionados son series de casos.

Sólo en 51 artículos primarios (82,2\%), se describen los órganos afectados a partir de los cuales se obtuvieron las muestras ( $\mathrm{n}=1.138)$; en los otros 11 artículos (373 muestras), no se reporta el origen anatómico de ellas. Las muestras provenían de hígado $(\mathrm{n}=802 ; 53,1 \%)$; pulmones $(\mathrm{n}=285 ; 18,9 \%)$; de otros órganos $(\mathrm{n}=51 ; 3,3 \%)$, entre los que destacan bazo, páncreas, riñones y peritoneo. Sin embargo, la información que relaciona órgano con genotipo sólo la entregan 10 artículos (16,1\%), que representan
Tabla 1. Estrategias de búsqueda utilizadas en las fuentes de información utilizadas y resultados obtenidos ( $\mathrm{n}: 701$ )

\begin{tabular}{|c|c|c|}
\hline \multicolumn{2}{|c|}{ Fuentes de información } & \multirow{2}{*}{$\begin{array}{l}\text { Estrategias de búsqueda } \\
\text { TI:((Echinococcosis* OR Echinococcus granulosus*) AND } \\
\text { (Genotype* OR Haplotypes* OR Molecular Epidemiology*)) }\end{array}$} \\
\hline WoS & $(n=90)$ & \\
\hline E & $(n=61)$ & $\begin{array}{l}\text { (echinococcosis:ti OR 'echinococcus granulosus':ti) AND } \\
\text { (genotype:ti OR haplotype:ti OR 'molecular epidemiology':ti) }\end{array}$ \\
\hline MEDI & $(n=288)$ & $\begin{array}{l}\text { (("Echinococcosis"[MeSH] OR "Echinococcus granulosus"[MeSH]) } \\
\text { AND ("Genotype"[MeSH] OR "Haplotypes"[MeSH] OR "Molecular } \\
\text { Epidemiology"[MeSH])) }\end{array}$ \\
\hline SCOP & $(n=83)$ & $\begin{array}{l}\text { TITLE (("Echinococcosis" OR "Echinococcus granulosus") AND } \\
\text { (genotype OR haplotype)) }\end{array}$ \\
\hline Trip Da & $(n=89)$ & $\begin{array}{l}\text { ("Echinococcosis" OR "Echinococcus granulosus")("Genotype" OR } \\
\text { "Haplotypes" OR "Molecular Epidemiology") }\end{array}$ \\
\hline BIREME-BVS & $(n=48)$ & $\begin{array}{l}\text { (ti:(Echinococcosis )) OR (ti:("Echinococcus granulosus")) AND } \\
\text { (ti:(Genotype OR Haplotypes OR “Molecular Epidemiology")) }\end{array}$ \\
\hline SCIELO & $(n=12)$ & $\begin{array}{l}\text { (Echinococcosis OR "Echinococcus granulosus") AND (Genotype } \\
\text { OR Haplotypes OR "Molecular Epidemiology") }\end{array}$ \\
\hline LILACS & $(n=11)$ & $\begin{array}{l}\text { (Echinococcosis OR "Echinococcus granulosus") AND (Genotype } \\
\text { OR Haplotypes OR "Molecular Epidemiology") }\end{array}$ \\
\hline IBECS & $(n=5)$ & $\begin{array}{l}\text { Echinoco } \$ \text { [Palabras del título] and Genotype [Palabras del título] } \\
\text { or Haplotype [Palabras del título] }\end{array}$ \\
\hline OPS-OMS & $(n=14)$ & Echinococcosis AND Genotype \\
\hline
\end{tabular}

WoS: Web of Science. EMBASE: Excerpta Medica dataBASE. BIREME: Biblioteca Regional de Medicina (Centro Latinoamericano y del Caribe de Información en Ciencias de la Salud). BVS: Biblioteca Virtual en Salud. IBECS: Índice Bibliográfico Español en Ciencias de la Salud. OPS-OMS: Organización Panemericana de la Salud - Organización Mundial de la Salud.

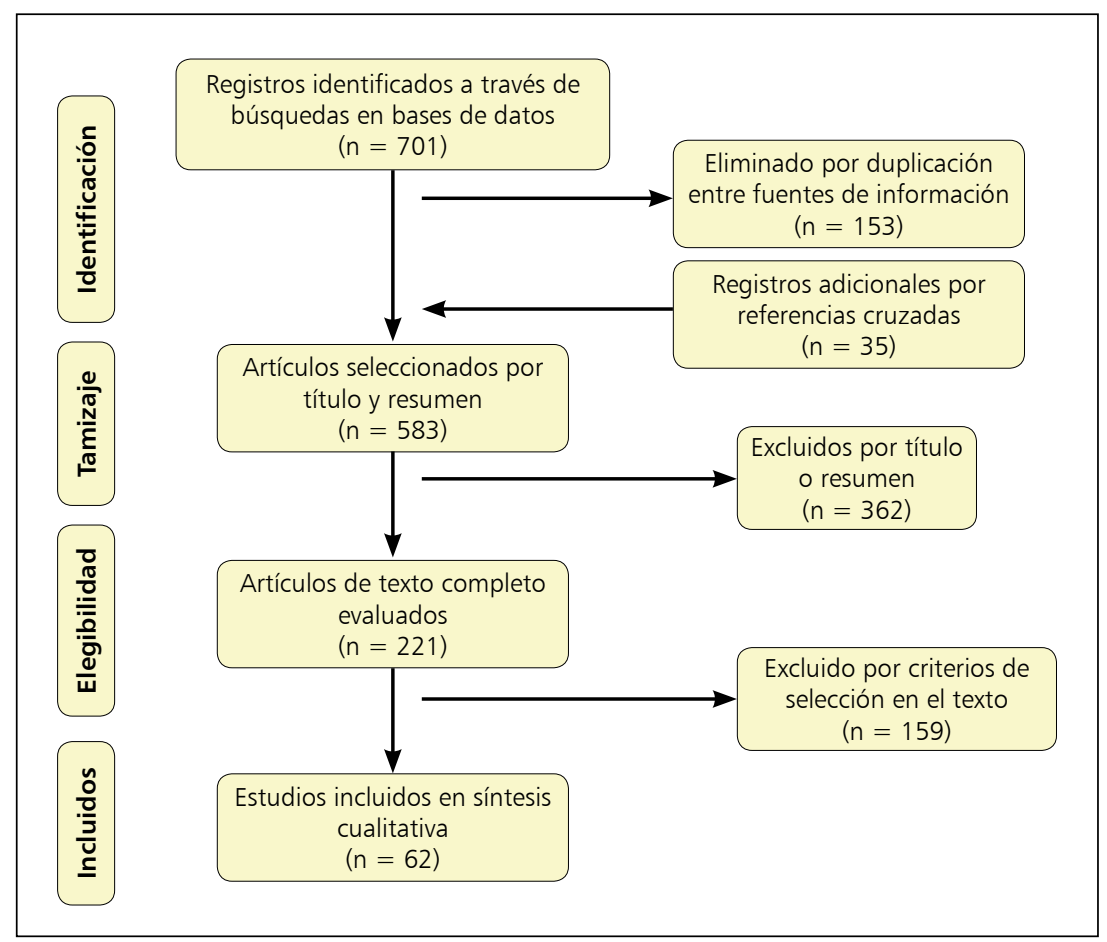

Figura 1. Flujograma de los estudios participantes (Moher et al., 2009). 


\begin{tabular}{|c|c|c|c|c|}
\hline Autor & $\mathrm{n}$ muestras & Hígado (n: 177) & Pulmones (n: 95) & Otros órganos (n: 15) \\
\hline Debiaggi ${ }^{17}$ & 21 & $\mathrm{G} 1=6 ; \mathrm{G} 6=5 ; \mathrm{G} 7=1$ & $\mathrm{G} 1=2 ; \mathrm{G} 6=4$ & $\mathrm{G} 1=1 ; \mathrm{G} 6=2$ \\
\hline Guarnera $^{26}$ & 41 & $\mathrm{G} 1=15 ; \mathrm{G} 2=4 ; \mathrm{G} 5=1 ; \mathrm{G} 6=9$ & $\mathrm{G} 1=3 ; \mathrm{G} 2=2 ; \mathrm{G} 6=5$ & $\mathrm{G} 1=1 ; \mathrm{G} 6=1$ \\
\hline Manterola ${ }^{39}$ & 20 & $\mathrm{G} 1=19 ; \mathrm{G} 6=1$ & --- & --- \\
\hline Mohaghegh $^{43}$ & 40 & $\mathrm{G} 1=10$ & $\mathrm{G} 1=22 ; \mathrm{G} 3=4 ; \mathrm{G} 6=1$ & $\mathrm{G} 1=3$ \\
\hline Nikmanesh $^{47}$ & 39 & $\mathrm{G} 1=11 ; \mathrm{G} 3=4$ & $\mathrm{G} 1=15 ; \mathrm{G} 3=2 ; \mathrm{G} 6=1$ & $\mathrm{G} 1=6$ \\
\hline Nikmanesh $^{48}$ & 30 & $\mathrm{G} 1=10 ; \mathrm{G} 3=3$ & $\mathrm{G} 1=15 ; \mathrm{G} 6=1$ & $\mathrm{G} 1=1$ \\
\hline Oral Babaoğlu ${ }^{49}$ & 20 & $\mathrm{G} 1=15 ; \mathrm{G} 6 / 7=5$ & --- & --- \\
\hline Orsten $^{50}$ & 46 & $\mathrm{G} 1=39 ; \mathrm{G} 3=7$ & --- & --- \\
\hline Santivañez ${ }^{57}$ & 20 & $\mathrm{G} 1=2$ & $\mathrm{G} 1=17 ; \mathrm{G} 6=1$ & \\
\hline Scott ${ }^{58}$ & 10 & $\mathrm{G} 9=10$ & --- & --- \\
\hline Total & 287 & $\begin{array}{l}\mathrm{G} 1=127 ; \mathrm{G} 2=4 ; \mathrm{G} 3=14 ; \mathrm{G} 5=1 ; \mathrm{G} 6=15 \\
\mathrm{G} 6 / 7=5 ; \mathrm{G} 7=1 ; \mathrm{G} 9=10\end{array}$ & $\mathrm{G} 1=74 ; \mathrm{G} 2=2 ; \mathrm{G} 3=6 ; \mathrm{G} 6=13$ & $\mathrm{G} 1=12 ; \mathrm{G} 6=3$ \\
\hline
\end{tabular}

sólo 287 muestras (19,0\%). Esta información, se resume en la Tabla 2.

La evidencia existente proviene principalmente de Irán, China, Argentina, Chile, Turquía y Perú con 28, 5, 4, 3, 3 y 3 estudios cada uno, respectivamente (Tablas 3 y 4).

El período con mayor número de artículos relacionados fue 2015-2019 (29/62 estudios; 46,8\%) (Tabla 5).

La técnica de secuenciación más utilizada fue Sanger, la cual incluye la amplificación de fragmentos de genes por medio de RPC. En la Tabla 6 se describen los genes secuenciados con mayor frecuencia en las muestras de los artículos primarios; destacando coxl y $\operatorname{nad} 1(79,0$ y $37,1 \%$, respectivamente).

Los genotipos identificados con mayor frecuencia fueron G1, el complejo G1/G3 y G6 (49,1\%; 32,2\% y $11,6 \%$ de las muestras estudiadas, respectivamente). No obstante, se reportó infección por los demás genotipos con frecuencias menores $(<3,5 \%)$; con excepción de $\mathrm{G} 4$, G8 y G10; de los cuales no se evidenció infección en los estudios incluidos en esta RS (Tabla 7).

Tabla 3. Resumen de la evidencia disponible

\begin{tabular}{|c|c|c|c|c|c|}
\hline Autor & Año & $\mathrm{n}$ muestras & Origen & Protocolos de secuenciación & Genotipos reportados \\
\hline Aaty ${ }^{8}$ & 2012 & 31 & Egipto & $r r n S$ & G6 \\
\hline Alvarez-Rojas $^{9}$ & 2017 & 13 & Chile & $\operatorname{cox} 1$ & G1, G2, G3 \\
\hline Arbabi10 & 2017 & 15 & Irán & $\operatorname{cox} 1$, nad1 & G1 \\
\hline Avila $^{11}$ & 2017 & 5 & Argentina & $\operatorname{cox} 1$ & G1, G5, G6-G7 \\
\hline Borji'12 & 2018 & 50 & Irán & its1 & G1/G3 \\
\hline Boufana $^{13}$ & 2015 & 4 & Reino Unido & $\operatorname{cox} 1$ & G1/G3 \\
\hline Boufana $^{14}$ & 2014 & 22 & Tunisia & cox1, ef1a & G1/G3 y G6 \\
\hline Bowles $^{15}$ & 1992 & 4 & Varios* & $\operatorname{cox} 1$ & G1 \\
\hline Casullii' & 2010 & 59 & Kenia & $\operatorname{cox} 1, \operatorname{nad} 1$ & G1 y G6 \\
\hline Daniel Mwambete ${ }^{17}$ & 2004 & 23 & España & $\operatorname{cox} 1, \operatorname{nad} 1$ & G1 y G7 \\
\hline Debiaggi ${ }^{17}$ & 2017 & 21 & Argentina & $\operatorname{cox} 1$, nad1 & G1, G6 y G7 \\
\hline De la Rue ${ }^{18}$ & 2011 & 6 & Brasil & cox $1, r r n s$ & G1, G3 y G5 \\
\hline Dousti ${ }^{19}$ & 2013 & 2 & Irán & its1 & G1/G3 \\
\hline Erdoğan²0 & 2017 & 25 & Turquía & $\operatorname{cox} 1$ & G1 \\
\hline Espinoza ${ }^{21}$ & 2014 & 3 & Chile & $\operatorname{cox} 1, \operatorname{nad} 1$ & G1 y G1/G3 \\
\hline Farhadi ${ }^{22}$ & 2015 & 9 & Irán & $\operatorname{cox} 1$ & G1 y G3 \\
\hline Gholami23 & 2012 & 30 & Irán & its1 & G1 y G6/G7 \\
\hline
\end{tabular}




\begin{tabular}{|c|c|c|c|c|c|}
\hline Autor & Año & $\mathrm{n}$ muestras & Origen & Protocolos de secuenciación & Genotipos reportados \\
\hline González²4 & 2002 & 2 & España & $\operatorname{cox} 1$, nad1 & G1 y G7 \\
\hline Gorgani-Firouzjaee ${ }^{25}$ & 2018 & 5 & Irán & cox1, its1 & G1/G3 \\
\hline Guarnera ${ }^{26}$ & 2004 & 41 & Argentina & $\operatorname{cox} 1$ & G1, G2, G5 y G6 \\
\hline $\mathrm{Guo}^{27}$ & 2019 & 26 & China & $\operatorname{cox} 1$ & G1 y G3 \\
\hline Hajialilo ${ }^{28}$ & 2012 & 1 & Irán & $\operatorname{cox} 1$, nad1 & G1 \\
\hline Hammad 29 & 2018 & 3 & Irak & $\operatorname{cox} 1, \mathrm{rrns}$ & $\mathrm{G} 1 / \mathrm{G} 3$ \\
\hline $\operatorname{Han}^{30}$ & 2019 & 93 & China & $\operatorname{cox} 1$ & G1/G3 \\
\hline Haniloo $^{31}$ & 2013 & 9 & Irán & its1 & G1/G3 \\
\hline Harandi ${ }^{32}$ & 2002 & 36 & Irán & its1 & G1/G3 y G6 \\
\hline Hasan $^{33}$ & 2016 & 4 & Irán & its 1, rrns & G1/G3 \\
\hline Jafari ${ }^{34}$ & 2018 & 50 & Irán & $\operatorname{cox} 1$, nad1 & G1, G3 y G6 \\
\hline Karamian ${ }^{35}$ & 2017 & 9 & Irán & cox1, nad1 & G1 y G6 \\
\hline Khademvatan ${ }^{36}$ & 2013 & 5 & Irán & its1 & G1 \\
\hline $\mathrm{Kia}^{37}$ & 2010 & 30 & Irán & its1 & G1 \\
\hline Konyaev $^{38}$ & 2013 & 3 & Rusia & $\operatorname{cox} 1$ & G1/G3 \\
\hline Manterola ${ }^{39}$ & 2008 & 20 & Chile & $\operatorname{cox} 1$ & G1/G3 y G6 \\
\hline Marinova ${ }^{40}$ & 2017 & 30 & Bulgaria & cox1, nad1, ef1a, hbx2, rrnL, rrnS, actll, atp6 & G1-G3 \\
\hline Matini $^{41}$ & 2018 & 9 & Irán & its1 & G1/G3 \\
\hline Matini $^{42}$ & 2019 & 10 & Irán & $\operatorname{cox} 1, \operatorname{nad} 1$ & G1 G2 y G3 \\
\hline Mohaghegh ${ }^{43}$ & 2019 & 40 & Irán & $\operatorname{cox} 1$ & G1, G3 y G6 \\
\hline Moro 44 & 2009 & 5 & Perú & $\operatorname{cox} 1$, nad1 & G1,G6 y G7 \\
\hline M'rad ${ }^{45}$ & 2005 & 11 & Túnez & cox1, its 1 & G1 y G6 \\
\hline $\mathrm{NakaO}^{46}$ & 2010 & 92 & China & cox1, ef1a & G1/G3 y G6 \\
\hline Nikmanesh ${ }^{47}$ & 2017 & 39 & Irán & cox1, nad1, actll, rrnS, atp6 & G1, G3 y G6 \\
\hline Nikmanesh ${ }^{48}$ & 2014 & 30 & Irán & $\operatorname{cox} 1$, nad1 & G1, G3 y G6 \\
\hline Oral Babaoğlư & 2018 & 20 & Turquía & $\operatorname{cox} 1$ & G1 y G6/G7 \\
\hline Orsten ${ }^{50}$ & 2018 & 46 & Turquía & $\operatorname{cox} 1$ & G1 y G3 \\
\hline Pezeshki ${ }^{51}$ & 2012 & 9 & Irán & $\operatorname{cox} 1$, nad1 & G1 y G3 \\
\hline Rosenzvit ${ }^{52}$ & 1999 & 9 & Argentina & cox1, nad1, its1 & $\mathrm{G} 1, \mathrm{G} 2, \mathrm{G} 6$ y G7 \\
\hline Rostami ${ }^{53}$ & 2015 & 125 & Irán & $\operatorname{cox} 1$ & G1, G2, G3 y G6 \\
\hline Sadjjadi ${ }^{4}$ & 2013 & 16 & Irán & $\operatorname{cox} 1$ & G1 y G6 \\
\hline Sałamatin ${ }^{55}$ & 2017 & 3 & Polonia & $\operatorname{cox} 1, \mathrm{rrnS}$ & G1 y G7 \\
\hline Sánchez ${ }^{56}$ & 2010 & 14 & Perú & $\operatorname{cox} 1$ & G1 \\
\hline Santivañez ${ }^{57}$ & 2008 & 20 & Perú & $\operatorname{cox} 1$ & G1 y G6 \\
\hline Scott 58 & 1997 & 10 & Polonia & nad1 & G9 \\
\hline Shahnazi ${ }^{59}$ & 2011 & 31 & Irán & cox1, nad1, its1 & G1 y G6 \\
\hline Shamsi ${ }^{60}$ & 2015 & 10 & Irán & nad1 & G1/G3 \\
\hline Shang ${ }^{61}$ & 2019 & 109 & China & $\operatorname{cox} 1$ & G1/G3 y G6/G7 \\
\hline Sharbatkhori62 & 2016 & 4 & Irán & $\operatorname{cox} 1$, nad1 & G1, G3 y G6 \\
\hline Spotin ${ }^{63}$ & 2016 & 41 & Irán & $\operatorname{cox} 1$ & G1, G2, G3 y G6 \\
\hline Tashani ${ }^{64}$ & 2002 & 3 & Libia & $\cos 1$ & G1 \\
\hline Vahedi ${ }^{65}$ & 2014 & 55 & Irán & its1 & G1 \\
\hline Zait $^{66}$ & 2016 & 54 & Argelia & $\operatorname{cox} 1$, nad1 & G1, G3 y G6 \\
\hline Zhang ${ }^{67}$ & 1998 & 3 & China & $\operatorname{cox} 1$, nad1 & G1 y G6 \\
\hline Zhang ${ }^{68}$ & 1998 & 4 & Irán & $\operatorname{cox} 1$, nad1 & G1 y G6 \\
\hline Total & & 1.511 & & & \\
\hline
\end{tabular}




\begin{tabular}{|lcc|}
\hline \multicolumn{3}{|c|}{ Tabla 4. Origen de los estudios analizados } \\
\hline Países & n estudios & $\%$ \\
\hline Irán & 28 & 45,2 \\
China & 5 & 8,1 \\
\hline Argentina & 4 & 6,5 \\
Chile & 3 & 4,8 \\
\hline Turquía & 3 & 4,8 \\
Perú & 3 & 4,8 \\
\hline España & 2 & 3,2 \\
\hline Polonia & 2 & 3,2 \\
\hline Túnez & 2 & 3,2 \\
Otros* & 10 & 16,2 \\
\hline Total & 62 & 100 \\
\hline *Argelia, Brasil, Bulgaria, Egipto, Irak, Kenia, Libia, Reino Unido, \\
Rusia, Muestras de varios países; con un estudio cada cual. \\
\hline
\end{tabular}

\section{Discusión}

Existen sólo tres RS en las que se reportan resultados referentes a caracterización genética de echinococcosis quística o hidatidosis. En una de ellas se analizan datos de artículos primarios con resultados en diferentes hospederos (humanos y animales, intermediarios y definitivos), como es el caso de Khademvatan (73 estudios, con 340 muestras humanas provenientes sólo de Irán) ${ }^{69}$. Otra, en la que sólo se analizaron estudios con muestras humanas provenientes de Irán (21 estudios, 559 muestras $)^{70}$.
Tabla 5. Distribución de los períodos de tiempo en que se publicaron los estudios analizados

\begin{tabular}{lcc}
\hline Período & n estudios & $\%$ \\
\hline $1990-1994$ & 1 & 1,66 \\
\hline $1995-1999$ & 4 & 6,4 \\
\hline $2000-2004$ & 5 & 8,1 \\
\hline $2005-2009$ & 4 & 6,4 \\
\hline $2010-2014$ & 19 & 30,6 \\
\hline $2015-2019$ & 29 & 46,8 \\
\hline Total & 62 & 100 \\
\hline
\end{tabular}

Tabla 6. Genes utilizados en la secuenciación de las muestras en los estudios analizados

\begin{tabular}{lcc}
\hline Genes utilizados* & n estudios & $\%$ \\
\hline cox1 & 49 & 79,0 \\
nad1 & 23 & 37,1 \\
\hline its1** & 15 & 24,2 \\
rrns & 7 & 11,2 \\
\hline ef1a** & 3 & 4,8 \\
\hline actll** & 2 & 3,2 \\
\hline atpP6** & 2 & 3,2 \\
\hline hbx2** & 1 & 1,6 \\
\hline rrnL & 1 & 1,6 \\
\hline
\end{tabular}

*En algunos estudios se utilizaron más de un gen para la realización de la genotipificación. **Genes nucleares (el resto de genes sin marca hacen referencia a genes mitocondriales).

Tabla 7. Genotipos encontrados en las muestras provenientes de los estudios analizados y en los diferentes estudios analizados

\begin{tabular}{|c|c|c|c|c|}
\hline Genotipos encontrados* & n de muestras & $\%$ & n de estudios* & $\%$ \\
\hline G1 & 742 & 49,1 & 43 & 69,4 \\
\hline $\mathrm{G} 2$ & 11 & 0,7 & 6 & 9,7 \\
\hline G3 & 50 & 3,3 & 15 & 24,2 \\
\hline $\mathrm{G} 1 / \mathrm{G} 3$ & 489 & 32,3 & 18 & 29,0 \\
\hline G4 & 0 & 0,0 & 0 & 0,0 \\
\hline G5 & 4 & 0,3 & 3 & 4,8 \\
\hline G6 & 175 & 11,6 & 25 & 40,3 \\
\hline G7 & 13 & 0,9 & 6 & 9,7 \\
\hline $\mathrm{G} 6 / \mathrm{G} 7$ & 17 & 1,1 & 4 & 6,5 \\
\hline G8 & 0 & 0,0 & 0 & 0,0 \\
\hline G9 & 10 & 0,7 & 1 & 1,6 \\
\hline G10 & 0 & 0,0 & 0 & 0,0 \\
\hline Total & 1.511 & 100 & & \\
\hline
\end{tabular}


algunos de los efectos que observamos. Por ejemplo, el hecho que exista un mayor número de publicaciones provenientes de Irán, no necesariamente significa que esa área sea más endémica que otras. Hay un claro infrareporte de esta situación en otras latitudes asociado a numerosas variables.

En conclusión, estos hallazgos mejoran nuestra comprensión acerca de la diversidad genética de E. granulosus. Sin embargo, es necesario implementar estudios exhaustivos para comprender mejor la caracterización morfo-cuantitativa del perfil genómico a través de la aplicación de diferentes enfoques bio-informáticos, de secuenciación de mitogenomas para una mayor resolución de los genotipos y clasificación, e identificación de nuevos candidatos diagnósticos.

A su vez, estos datos aumentan el potencial para identificar vacunas y tratamientos dianas; pueden ayudar en el desarrollo y estandarización de técnicas de biología molecular para la identificación de regiones en el genoma y/o proteínas de utilidad para el diagnóstico selectivo y sensible de la infección por E. granulosus. Sin embargo, dado que G1 y el complejo G1/G3 son los más prevalentes en humanos, las iniciativas de control de hidatidosis deben dirigirse especialmente al hospedero definitivo de este ciclo (perros) y a sus hospederos intermediarios (ganado bovino, ovino y caprino).

\section{Referencias bibliográficas}

1.- Agudelo Higuita N I, Brunetti E, McCloskey C. Cystic echinococcosis. J Clin Microbiol 2016 54 (3): 518-23. doi: 10.1128/JCM.02420-15.

2.- da Silva A M. Human echinococcosis: a neglected disease. Gastroenterol Res Pract. 2010; 2010. doi: 10.1155/2010/583297.

3.- Bhutani N, Kajal P. Hepatic echinococcosis: A review. Ann Med Surg (Lond) 2018; 36: 99105. doi: 10.1016/j.amsu.2018.10.032.

4.- Budke C M, Deplazes P, Torgerson P R. Global socioeconomic impact of cystic echinococcosis. Emerg Infect Dis 2006; 12 (2): 296-303. doi: 10.3201/eid1202.050499.

5.- Manterola C, Otzen T. Molecular epidemiology of cystic echinococcosis: Genotypic characterization in humans and different livestock. Int J Morphol 2016; 34(4): 1472-81. doi: 10.4067/S0717-95022016000400048.

6.- Daniel Mwambete K, Ponce-Gordo F, CuestaBandera C. Genetic identification and host range of the Spanish strains of Echinococcus granulosus. Acta Trop 2004; 91 (2): 87-93. doi: 10.1016/j.actatropica.2004.04.001

7.- $\quad$ Moher D, Liberati A, Prisma Group. Preferred reporting items for systematic reviews and meta-analyses: the PRISMA Statement. Ann Intern Med 2009; 151 (4): 264-9. doi: 10.7326/0003-4819-151-4-20090818000135 .

8.- $\quad$ Aaty H E, Abdel-Hameed D M, Alam-Eldin Y H, El-Shennawy S F, Aminou H A, Makled S $\mathrm{S}$, et al. Molecular genotyping of E. granulosus in animal and human isolates from Egypt. Acta Trop 2012; 121: 125-8. doi: 10.1016/j. actatropica.2011.10.014.

9.- Alvarez Rojas C A, Ebi D, Paredes R, Acosta-Jamett G, Urriola N, Roa J C, et al. High intraspecific variability of Echinococcus granulosus sensu stricto in Chile. Parasitol Int 2017; 66 (2): 112-5. doi: 10.1016/j. parint.2016.12.001.

10.- Arbabi M, Pirestani M, Delavari M, Hooshyar H, Abdoli A, Sarvi S. Molecular and morphological characterizations of Echinococcus granulosus from human and animal isolates in Kashan, Markazi Province, Iran. Iran J Parasitol 2017; 12(2): 177-87. PMID: 28761477.

11.- Avila H G, Santos G B, Cucher M A, Macchiaroli N, Pérez M G, Baldi G, et al. Implementation of new tools in molecular epidemiology studies of Echinococcus granulosus sensu lato in South America. Parasitol Int 2017; 66: 250-7. doi: 10.1016/j. parint.2017.02.001.

12.- Borji H, Bahar M M, Naghibi A, Masoom S H
F. Genotyping of human cystic echinococcosis in northeastern Iran. Ann Parasitol 2018; 64 (4): 323-9. doi: 10.17420/ap6404.167.

13.- Boufana B, Lett W S, Lahmar S, Buishi I, Bodell A J, Varcasia A, et al. Echinococcus equinus and Echinococcus granulosus sensu stricto from the United Kingdom: genetic diversity and haplotypic variation. Int $\mathrm{J}$ Parasitol 2015; 45: 161-6. doi: 10.1016/j. ijpara.2014.10.005.

14.- Boufana B, Lahmar S, Rebaï W, Ben Safta Z, Jebabli L, Ammar A, et al. Genetic variability and haplotypes of Echinococcus isolates from Tunisia. Trans R Soc Trop Med Hyg 2014; 108(11): 706-14. doi: 10.1093/trstmh/tru138.

15.- Bowles J, Blair D, McManus D P. Genetic variants within the genus Echinococcus identified by mitochondrial DNA sequencing. Mol Biochem Parasitol 1992; 54 (2): 165-73. doi: 10.1016/0166-6851(92)90109-W.

16.- Casulli A, Zeyhle E, Brunetti E, Pozio E, Meroni V, Genco F, Filice C. Molecular evidence of the camel strain (G6 genotype) of E. granulosus in humans from Turkana, Kenya Trans R Soc Trop Med Hyg 2010; 104: 29-32. doi: 10.1016/j.trstmh.2009.08.001.

17.- Debiaggi M F, Soriano S V, Pierangeli N B, Lazzarini L E, Pianciola L A, Mazzeo M L, et al. Genetic characterization of human hydatid 
cysts shows coinfection by Echinococcus canadensis $\mathrm{G} 7$ and E. granulosus sensu stricto G1 in Argentina. Parasitol Res 2017; 116: 2599-604. doi: 10.1007/s00436-017-5555-2.

18.- De la Rue M L, Takano K, Brochado J F, Costa C V, Soares A G, Yamano K, et al. Infection of humans and animals with Echinococcus granulosus (G1 and G3 strains) and E. ortleppi in Southern Brazil. Vet Parasitol 2011; 177: 97 103. doi: 10.1016/j.vetpar.2010.11.018.

19.- Dousti M, Abdi J, Bakhtiyari S, Mohebali M, Mirhendi S, Rokni M. Genotyping of hydatid cyst isolated from human and domestic animals in Ilam Province, Western Iran using PCR-RFLP. Iran J Parasitol 2013; 8: 47-52. PMID: 23682259.

20.- Erdoğan E, Özkan B, Mutlu F, Karaca S, Şahin İ. Molecular characterization of E. granulosus isolates obtained from different hosts. Mikrobiyol Bul 2017; 51: 79-86. doi: 10.5578/ mb.45452.

21.- Espinoza S, Salas A M, Vargas A, Freire V, Díaz E, Sánchez G, et al. Detection of the G3 genotype of Echinococcus granulosus from hydatid cysts of Chilean cattle using COX1 and ND1 mitochondrial markers. Parasitol Res 2014; 113: 139-47. doi: 10.1007/s00436-0133636-4.

22.- Farhadi M, Fazaeli A, Haniloo A. Genetic characterization of livestock and human hydatid cyst isolates from northwest Iran, using the mitochondrial cox1 gene sequence. Parasitol Res. 2015; 114: 4363-70. doi: 10.1007/s00436015-4673-y.

23.- Gholami Sh, Sosari M, Fakhar M, Sharif M, Daryani A, Hashemi M, et al. Molecular characterization of Echinococcus granulosus from hydatid cysts isolated from human and animals in Golestan Province, North of Iran. Iran J Parasitol 2012; 7 (4): 8-16. PMID: 23323086.

24.- González L M, Daniel-Mwambete K, Montero E, Rosenzvit M C, McManus D $\mathrm{P}$, Gárate T, Cuesta-Bandera C. Further molecular discrimination of Spanish strains of Echinococcus granulosus. Exp Parasitol 2002; 102: 45-56. doi: 10.1016/s00144894(02)00146-7.

25.- Gorgani-Firouzjaee T, Kalantrai N, Ghaffari S, Alipour J, Siadati S. Genotype characterization of livestock and human cystic echinococcosis in Mazandaran Province, Iran. J Helminthol 2018; 93(2): 255-9. doi: 10.1017/ S0022149X1800010X.

26.- Guarnera E A, Parra A, Kamenetzky L, García G, Gutiérrez A. Cystic echinococcosis in Argentina: evolution of metacestode and clinical expression in various Echinococcus granulosus strains. Acta Trop 2004; 92: 153-9. doi: 10.1016/j.actatropica.2004.06.008.

27.- Guo B, Zhang Z, Zheng X, Guo Y, Guo G, Zhao L, et al. Prevalence and molecular characterization of Echinococcus granulosus sensu stricto in Northern Xinjiang, China. Korean J Parasitol 2019; 57 (2): 153-9. doi: 10.3347/kjp.2019.57.2.153.

28.- Hajialilo E, Harandi M F, Sharbatkhori M, Mirhendi H, Rostami S. Genetic characterization of Echinococcus granulosus in camels, cattle and sheep from the south-east of Iran indicates the presence of the G3 genotype. J Helminthol 2012; 86: 263-70. doi: 10.1017/ S0022149X11000320.

29.- Hammad S J, Cavallero S, Milardi G L, Gabrielli S, D Amelio S, Al-Nasiri F S. Molecular genotyping of Echinococcus granulosus in the North of Iraq. Vet Parasitol 2018; 249: 82-7. doi: 10.1016/j. vetpar.2017.11.010.

30.- Han X, Jian Y, Zhang X, Ma L, Zhu W, Cai Q, et al. Genetic characterization of Echinococcus isolates from various intermediate hosts in the Qinghai-Tibetan Plateau Area, China. Parasitology 2019; 146 (10): 1305-12. doi: $10.1017 / \mathrm{S} 0031182019000544$

31.- Haniloo A, Farhadi M, Fazaeli A, Nourian N. Genotype characterization of hydatid cysts isolated from Zanjan using PCR-RFLP technique. J Zanjan Univ Med Sci Health Services 2013; 21: 57-65. https://www.magiran. com/paper/1096420?lang=en.

32.- Harandi M F, Hobbs R P, Adams P J, Mobedi I, Morgan-Ryan U M, Thompson R C A. Molecular and morphological characterization of Echinococcus granulosus of human and animal origin in Iran. Parasit ology 2002; 125 : 367-73. doi: 10.1017/s0031182002002172.

33.- Hassan H F, Fadhil M H, Fadhil Z H. Molecular characterization of Echinococcus granulosus isolated from human and domestic animals in Kirkuk, Iraq. Animal Res International 2016; 13 (3): 2544-7. https://www.ajol.info/index. php/ari/article/view/149940.

34.- Jafari R, Sanei B, Baradaran A, Spotin A, Bagherpour B, Darani H Y. Genetic characterization of Echinococcus granulosus strains isolated from humans based on nad1 and cox 1 gene analysis in Isfahan, central Iran. J Helminthol 2018; 92 (6): 696-702. doi: 10.1017/S0022149X17000967.

35.- Karamian M, Haghighi F, Hemmati M, Taylor WR, Salehabadi A, Ghatee MA. Heterogenity of Echinococcus canadensis genotype 6 - the main causative agent of cystic echinococcosis in Birjand, Eastern Iran. Vet Parasitol 2017; 245: 78-85. doi: 10.1016/j.vetpar.2017.08.018.

36.- Khademvatan S, Yousefi E, Rafiei A, Rahdar M, Saki J. Molecular characterization of livestock and human isolates of Echinococcus granulosus from south-west Iran. J Helminthol 2013; 87 (2): 240-4. doi: 10.1017/S0022149X12000296.

37.- Kia E B, Rahimi H, Sharbatkhori M, Talebi A, Fasihi Harandi M, Mirhendi H. Genotype identification of human cystic echinococcosis in Isfahan, central Iran. Parasitol Res 2010; 107 (3): 757-60. doi: 10.1007/s00436-010-1947-2.

38.- Konyaev S V, Yanagida T, Nakao M, Ingovatova $\mathrm{G} \mathrm{M}$, Shoykhet $\mathrm{Y} \mathrm{N}$, Bondarev A Y, et al. Genetic diversity of Echinococcus spp. in Russia. Parasitology 2013; 140: 1637-47. doi: 10.1017/S0031182013001340.

39.- Manterola C, Benavente F, Melo A, Vial M, Roa J C. Description of Echinococcus granulosus genotypes in human hydatidosis in a region of southern Chile. Parasitol Int 2008; 57 (3): 342-6. doi: 10.1016/j.parint.2008.02.005.

40.- Marinova I, Spiliotis M, Wang J, Muhtarov M, Chaligiannis I, Sotiraki S, et al. Molecular characterization of Echinococcus granulosus isolates from Bulgarian human cystic echinococcosis patients. Parasitol Res 2017; 116: 1043-54. doi: 10.1007/s00436-017-53861.

41.- Matini M, Roostaei M, Fallah M, Maghsood A H, Saidijam M, Fasihi Harandi M. Genetic identification of Echinococcus granulosus isolates in Hamadan, Western Iran. Iran J Parasitol 2018; 13 (3): 423-9.

42.- Matini M, Fallah M, Maghsood AH, Saidijam M, Fasihi Harandi M. Echinococcus granulosus sensu stricto in livestock and human in Hamadan, Western Iran. Iran J Parasitol 2019; 14 (2): 288-96. PMID: 31543917.

43.- Mohaghegh M A, Yousofi-Darani H, Jafarian A H, Mirbadie S R, Fasihi-Harandi M, Ghavimi R, et al. Isolated human and livestock Echinococcus granulosus genotypes using real-time PCR of cox1 gene in Northeast Iran. Acta Parasitol 2019; 64: 679-85. doi: 10.2478/ s11686-019-00117-w.

44.- Moro P L, Nakao M, Ito A, Schantz P M, Cavero C, Cabrera L. Molecular identification of Echinococcus isolates from Peru. Parasitol Int 2009; 58: 184-6. doi: 10.1016/j. parint.2009.01.005.

45.- M'rad S, Filisetti D, Oudni M, Mekki M, Belguith M, Nouri A, et al. Molecular evidence of ovine (G1) and camel (G6) strains of $E$. granulosus in Tunisia and putative role of cattle in human contamination. Vet Parasitol 2005; 129: 267-72. doi: 10.1016/j.vetpar.2005.02.006.

46.- Nakao M, Li T, Han X, Ma X, Xiao N, Qiu J, et al. Genetic polymorphisms of Echinococcus tapeworms in China as determined by mitochondrial and nuclear DNA sequences. Int J Parasitol 2010; 40: 379-85. doi: 10.1016/j. ijpara.2009.09.006.

47.- Nikmanesh B, Mirhendi H, Mahmoudi S, Rokni MB. Multilocus sequence analysis of Echinococcus granulosus strains isolated from humans and animals in Iran. Exp Parasitol 2017; 183: 50-5. doi: 10.1016/j. exppara.2017.10.002.

48.- Nikmanesh B, Mirhendi H, Ghalavand Z, Alebouyeh M, Sharbatkhori M, Kia E, et al. Genotyping of Echinococcus granulosus 
isolates from human clinical samples based on sequencing of mitochondrial genes in Iran, Tehran. Iran J Parasitol. 2014; 9: 20-7. PMID: 25642256.

49.- Oral Babaoğlu A, Soyder A, Malatyalı E, Ertuğ S, Ertabaklar H. Genotyping of Echinococcus granulosus isolates by sequencing of mitochondrial cytochrome $\mathrm{C}$ oxidase subunit 1 (cox1) gene in Aydin. Mikrobiyol Bul 2018; 52 (2): 198-205. doi: 10.5578/mb.66711.

50.- Orsten S, Boufana B, Ciftci T, Akinci D, Karaagaoglu E, Ozkuyumcu C, et al. Human cystic echinococcosis in Turkey: a preliminary study on DNA polymorphisms of hydatid cysts removed from confirmed patients. Parasitol Res 2018; 117 (4): 1257-63. doi: 10.1007/s00436018-5807-9.

51.- Pezeshki A, Akhlaghi L, Sharbatkhori M, Razmjou E, Oormazdi H, Mohebali M, et al. Genotyping of Echinococcus granulosus from domestic animals and humans from Ardabil Province, northwest Iran. J Helminthol 2013; 87: 387-91. doi: 10.1017/ S0022149X1200051X.

52.- Rosenzvit M, Zhang L-H, Kamenetzky L, Canova S, Guarnera E, McManus D. Genetic variation and epidemiology of Echinococcus granulosus in Argentina. Parasitology 1999; 118 (Pt 5): 523-30. doi: 10.1017/ s0031182099004035.

53.- Rostami S, Torbaghan S S, Dabiri S, Babaei Z, Mohammadi M A, Sharbatkhori M, et al. Genetic characterization of Echinococcus granulosus from a large number of formalin fixed, paraffin-embedded tissue samples of human isolates in Iran. Am J Trop Med Hyg 2015; 92: 588-94. doi: 10.4269/ajtmh.14-0585.

54.- Sadjjadi S M, Mikaeili F, Karamian M, Maraghi S, Sadjjadi FS, Shariat-Torbaghan $\mathrm{S}$, et al. Evidence that the Echinococcus granulosus G6 genotype has an affinity for the brain in humans. Int J Parasitol 2013; 43 (11): 875-7. doi: 10.1016/j.ijpara.2013.06.008.

55.- Sałamatin R, Kowal J, Nosal P, Kornaś S, Cielecka D, Jańczak D, et al. Cystic echinococcosis in Poland: genetic variability and the first record of Echinococcus granulosus (G1 genotype) in the country. Parasitol Res 2017; 116: 3077-85. doi: 10.1007/s00436-017-
5618-4.

56.- Sánchez E, Cáceres O, Náquira C, García D, Patiño G, Silvia H, et al. Molecular characterization of Echinococcus granulosus from Peru by sequencing of the mitochondrial cytochrome $\mathrm{C}$ oxidase subunit 1 gene. Mem Inst Oswaldo Cruz 2010; 105 (6): 806-10. doi: 10.1590/s0074-02762010000600013.

57.- Santivañez S J, Gutierrez A M, Rosenzvit M C, Muzulin P M, Rodriguez M L, Vasquez J C, et al. Human hydatid disease in Peru is basically restricted to E. granulosus genotype G1. Am J Trop Med Hyg 2008; 79: 89-92. doi: 10.4269/ ajtmh.2008.79.89.

58.- Scott J C, Stefaniak J, Pawlowski Z S, McManus D P. Molecular genetic analysis of human cystic hydatid cases from Poland: identification of a new genotypic group (G9) of Echinococcus granulosus. Parasitology 1997; 114: 37-43. doi: 10.1017/s0031182096008062.

59.- Shahnazi M, Hejazi H, Salehi M, Andalib A R. Molecular characterization of human and animal Echinococcus granulosus isolates in Isfahan, Iran. Acta Trop 2011; 117 (1): 47-50. doi: 10.1016/j.actatropica.2010.09.002.

60.- Shamsi M, Dalimi A, Khosravi A, Ghafarifar F. Determination of genotype isolates of human and sheep hydatid cyst in Ilam. J Ilam Univ Med Sci 2015; 23 (2): 111-9.

61.- Shang J, Zhang G, Yu W, He W, Wang Q, Zhong B, et al. Molecular characterization of human echinococcosis in Sichuan, Western China. Acta Trop 2019; 190: 45-51. doi: 10.1016/j.actatropica.2018.09.019.

62.- Sharbatkhori M, Tanzifi A, Rostami S, Rostami M, Fasihi Harandi M. Echinococcus granulosus sensu lato genotypes in domestic livestock and humans in golestan province, Iran. Rev Inst Med Trop Sao Paulo 2016; 58: 38. doi: 10.1590/S1678-9946201658038.

63.- Spotin A, Mahami-Oskouei M, Harandi MF, Baratchian M, Bordbar A, Ahmadpour E, et al. Genetic variability of E. granulosus complex in various geographical populations of Iran inferred by mitochondrial DNA sequences. Acta Trop 2017; 165: 10-6. doi: 10.1016/j. actatropica.2016.03.002

64.- Tashani O, Zhang L H, Boufana B, Jegi A, McManus D P. Epidemiology and strain characteristics of Echinococcus granulosus in the Benghazi area of eastern Libya. Ann Trop Med Parasitol 2002; 96(4): 369-81. doi: $10.1179 / 000349802125000952$.

65.- Vahedi A, Mahdavi M, Ghazanchaei A, Shokouhi B. Genotypic characteristics of hydatid cysts isolated from humans in East Azerbaijan Province (2011-2013). J Anal Res Clin Med 2014; 2 (3): 152-7. doi: 10.5681/ jarcm.2014.025.

66.- Zait H, Kouidri M, Grenouillet F E, Umhang G, Millon L, Hamrioui B, et al. Molecular characterization of Echinococcus granulosus sensu stricto and Echinococcus canadensis in humans and livestock from Algeria. Parasitol Res 2016; 115: 2423-31. doi: 10.1007/s00436016-4994-5.

67.- Zhang L H, Chai J J, Jiao W, Osman Y, McManus D P. Mitochondrial genetic markers confirm the presence of the camel strain (G6 genotype) of Echinococcus granulosus in north-western China. Parasitology 1998; 116: 29-33. doi: 10.1017/s0031182097001881.

68.- Zhang L, Eslami A, Hosseini S H, McManus D P. Indication of the presence of two distinct strains of Echinococcus granulosus in Iran by mitochondrial DNA markers. Am J Trop Med Hyg 1998; 59: 171-4. doi: 10.4269/ ajtmh.1998.59.171.

69.- Khademvatan S, Majidiani H, Foroutan M, Hazrati Tappeh K, Aryamand S, Khalkhali H R. Echinococcus granulosus genotypes in Iran: a systematic review. J Helminthol. 2019; 93 (2): 131-8. doi: 10.1017/S0022149X18000275.

70.- Siyadatpanah A, Anvari D, Emami Zeydi A, Hosseini S A, Daryani A, Sarvi S, et al. A systematic review and meta-analysis of the genetic characterization of human echinococcosis in Iran, an endemic country. Epidemiol Health 2019; 41: e2019024. doi: 10.4178/epih.e2019024.

71.- Cucher M A, Macchiaroli N, Baldi G, Camicia F, Prada L, Maldonado L, et al. Cystic echinococcosis in South America: systematic review of species and genotypes of Echinococcus granulosus sensu lato in humans and natural domestic hosts. Trop Med Int Health 2016; 21 (2): 166-75. doi: 10.1111/ tmi. 12647 . 